Internat. J. Math. \& Math. Sci

Vol. 23, No. 1 (2000) 1-9

S0161171200001782

(C) Hindawi Publishing Corp.

\title{
ANALOGUES OF SOME FUNDAMENTAL THEOREMS OF SUMMABILITY THEORY
}

\author{
RICHARD F. PATTERSON
}

(Received 18 February 1998)

\begin{abstract}
In 1911, Steinhaus presented the following theorem: if $A$ is a regular matrix then there exists a sequence of 0's and 1's which is not $A$-summable. In 1943, R. C. Buck characterized convergent sequences as follows: a sequence $x$ is convergent if and only if there exists a regular matrix $A$ which sums every subsequence of $x$. In this paper, definitions for "subsequences of a double sequence" and "Pringsheim limit points" of a double sequence are introduced. In addition, multidimensional analogues of Steinhaus' and Buck's theorems are proved.
\end{abstract}

Keywords and phrases. Subsequences of a double sequence, Pringsheim limit point, P-convergent, P-divergent, RH-regular.

2000 Mathematics Subject Classification. Primary 40B05.

1. Introduction. In $[2,3,4,5,8]$, the 4-dimensional matrix transformation $(A x)_{m, n}=$ $\sum_{k, l=0,0}^{\infty, \infty} a_{m, n, k, l} x_{k, l}$ is studied extensively by Robison and Hamilton. Here we define new double sequence spaces and consider the behavior of 4-dimensional matrix transformations on our new spaces. Such a 4-dimensional matrix $A$ is said to be RH-regular if it maps every bounded P-convergent sequence (defined below) into a P-convergent sequence with the same P-limit. In [9] Steinhaus proved the following theorem: if $A$ is a regular matrix then there exists a sequence of 0's and 1's which is not $A$-summable. This implies that $A$ cannot sum every bounded sequence. In this paper, we prove a theorem for double sequences and 4-dimensional RH-regular matrices that is analogous to Steinhaus' theorem. One of the fundamental facts of sequence analysis is that if a sequence is convergent to $L$, then all of its subsequences are convergent to $L$. In a similar manner, R. C. Buck [1] characterized convergent sequences by: a sequence $x$ is convergent if and only if there exists a regular matrix $A$ which sums every subsequence of $x$. We characterize P-convergent double sequences as follows: first, we prove that a double sequence $x$ is P-convergent to $L$ if all of its subsequences are Pconvergent to $L$; then we prove that a double sequence $x$ is P-convergent if there exists an RH-regular matrix $A$ which sums every subsequence of $x$. In addition, we provide definitions for "subsequences" and "Pringsheim limit points" of double sequences and for divergent double sequence.

\section{Definitions, notations, and preliminary results}

Definition 2.1 (Pringsheim, 1900). A double sequence $x=\left[x_{k, l}\right]$ has Pringsheim limit $L$ (denoted by P-lim $x=L$ ) provided that given $\epsilon>0$ there exists $N \in \mathbb{N}$ such that 
$\left|x_{k, l}-L\right|<\epsilon$ whenever $k, l>N$. We describe such an $x$ more briefly as "P-convergent."

DEFINITION 2.2 (Pringsheim, 1900). A double sequence $x$ is called definite divergent, if for every (arbitrarily large) $G>0$ there exist two natural numbers $n_{1}$ and $n_{2}$ such that $\left|x_{n, k}\right|>G$ for $n \geq n_{1}, k \geq n_{2}$.

DEFINITION 2.3. The sequence $y$ is a subsequence of the double sequence $x$ provided that there exist two increasing double index sequences $\left\{n_{j}^{i}\right\}$ and $\left\{k_{j}^{i}\right\}$ such that $n_{0}^{1}=k_{0}^{1}=n_{-1}^{0}=k_{-1}^{0}=0$ and

$n_{1}^{i}$ and $k_{1}^{i}$ are both chosen such that $\max \left\{n_{2 i-3}^{i-1}, k_{2 i-3}^{i-1}\right\}<n_{1}^{i}, k_{1}^{i}$,

$n_{2}^{i}$ and $k_{2}^{i}$ are both chosen such that $\max \left\{n_{1}^{i}, k_{1}^{i}\right\}<n_{2}^{i}, k_{2}^{i}$,

$n_{3}^{i}$ and $k_{3}^{i}$ are both chosen such that $\max \left\{n_{2}^{i}, k_{2}^{i}\right\}<n_{3}^{i}, k_{3}^{i}$,

:

$n_{2 i-1}^{i}$ and $k_{2 i-1}^{i}$ are both chosen such that $\max \left\{n_{2(i-1)}^{i}, k_{2(i-1)}^{i}\right\}<n_{2 i-1}^{i}, k_{2 i-1}^{i}$, with

$$
\begin{aligned}
& y_{1, i}=x_{n_{1}^{i}, k_{1}^{i}}, \quad y_{2, i}=x_{n_{2}^{i}, k_{2}^{i}}, \quad y_{3, i}=x_{n_{3}^{i}, k_{3}^{i}}, \\
& \vdots \\
& y_{i, i}=x_{n_{i}^{i}, k_{i}^{i}}, \quad y_{i, i-1}=x_{n_{i+1}^{i}, k_{i+1}^{i}}, \\
& \vdots \\
& y_{i, 2 i-1}=x_{n_{2 i-1}^{i}, k_{2 i-1}^{i}}
\end{aligned}
$$

for $i=1,2,3, \ldots$.

A double sequence $x$ is bounded if and only if there exists a positive number $M$ such that $\left|x_{k, l}\right|<M$ for all $k$ and $l$. Define

$$
\begin{aligned}
S^{\prime \prime}\{x\} & =\{\text { all subsequences of } x\} ; \\
C^{\prime \prime} & =\{\text { all bounded P-convergent sequences }\} \\
C_{A}^{\prime \prime} & =\left\{x_{k, l}:(A x)_{m, n}=\sum_{k, l=0,0}^{\infty, \infty} a_{m, n, k, l} x_{k, l} \text { is P-convergent }\right\} .
\end{aligned}
$$

See Figure 1 for an illustration of the procedure for selecting terms of a subsequence. A 2-dimensional matrix transformation is said to be regular if it maps every convergent sequence into a convergent sequence with the same limit. The Silverman-Toeplitz theorem [6] characterizes the regularity of 2-dimensional matrix transformations. In 1926, Robison presented a 4-dimensional analog of regularity for double sequences in which he added an additional assumption of boundedness. This assumption was made because a double sequence which is P-convergent is not necessarily bounded. The definition of the regularity for 4-dimensional matrices will be stated below, with the Robison-Hamilton characterization of the regularity of 4-dimensional matrices. 


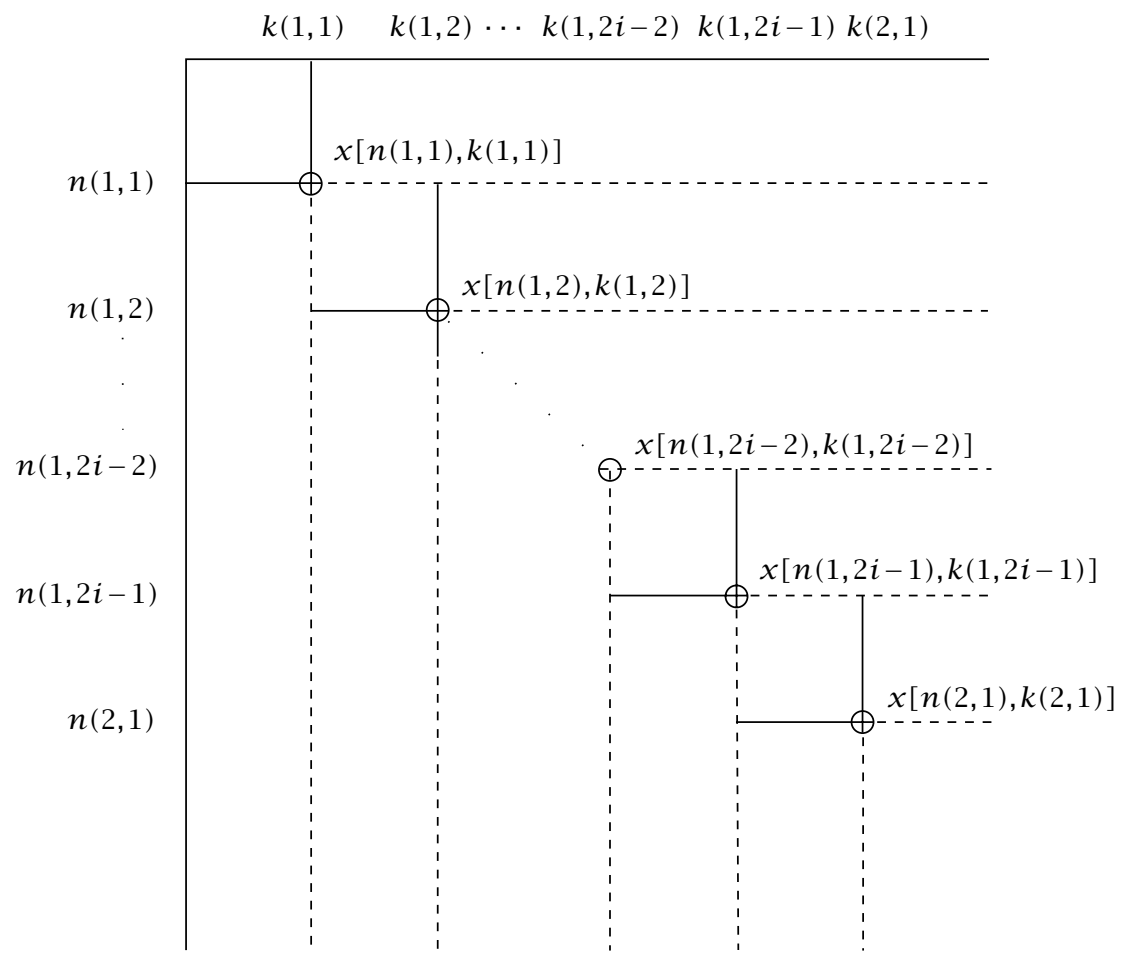

FIGURE 1. The selection process of terms for subsequence $y$ of $x$, where $x[n(i, j), k(i, j)]=x_{n_{j}^{i}, k_{j}^{i}}, n(i, j)=n_{j}^{i}, k(i, j)=k_{j}^{i}$.

DEFINITION 2.4. The 4-dimensional matrix $A$ is said to be RH-regular if it maps every bounded P-convergent sequence into a P-convergent sequence with the same P-limit.

THEOREM 2.1 (Hamilton [2], Robison [8]). The 4-dimensional matrix A is RH-regular if and only if

$\mathrm{RH}_{1}: \mathrm{P}-\lim _{m, n} a_{m, n, k, l}=0$ for each $k$ and $l$;

$\mathrm{RH}_{2}: \mathrm{P}-\lim _{m, n} \sum_{k, l=0,0}^{\infty} a_{m, n, k, l}=1$;

$\mathrm{RH}_{3}: \mathrm{P}-\lim _{m, n} \sum_{k=0}^{\infty}\left|a_{m, n, k, l}\right|=0$ for each $l$;

$\mathrm{RH}_{4}: \mathrm{P}-\lim _{m, n} \sum_{l=0}^{\infty}\left|a_{m, n, k, l}\right|=0$ for each $k$;

$\mathrm{RH}_{5}: \sum_{k, l=0,0}^{\infty, \infty}\left|a_{m, n, k, l}\right|$ is $\mathrm{P}$-convergent;

$\mathrm{RH}_{6}$ : there exist finite positive integers $A$ and $B$ such that $\sum_{k, l>B}\left|a_{m, n, k, l}\right|<A$.

REMARK 2.1. The definition of a Pringsheim limit point can also be stated as follows: $\beta$ is a Pringsheim limit point of $x$ provided that there exist two increasing index sequences $\left\{n_{i}\right\}$ and $\left\{k_{i}\right\}$ such that $\lim _{i} x_{n_{i}, k_{i}}=\beta$.

Definition 2.5. A double sequence $x$ is divergent in the Pringsheim sense (Pdivergent) provided that $x$ does not converge in the Pringsheim sense (P-convergent). 
REMARK 2.2. Definition 2.5 can also be stated as follows: a double sequence $x$ is P-divergent provided that either $x$ contains at least two subsequences with distinct finite limit points or $x$ contains an unbounded subsequence. Also note that, if $x$ contains an unbounded subsequence then $x$ also contains a definite divergent subsequence.

REMARK 2.3. For an ordinary single-dimensional sequence, any sequence is a subsequence of itself. This, however, is not the case in the 2-dimensional plane, as illustrated by the following example.

EXAMPLE 2.1. The sequence

$$
x_{n, k}:= \begin{cases}1, & \text { if } n=k=0, \\ 1, & \text { if } n=0, k=1, \\ 1, & \text { if } n=1, k=0, \\ 0, & \text { otherwise }\end{cases}
$$

contains only two subsequences, namely, $\left[y_{n, k}\right]=0$ for each $n$ and $k$, and

$$
z_{n, k}:= \begin{cases}1, & \text { if } n=k=0 \\ 0, & \text { otherwise }\end{cases}
$$

neither subsequence is $x$.

The following proposition is easily verified, and is worth stating since each singledimensional sequence is a subsequence of itself. However, this is not the case for double-dimensional sequences.

Proposition 2.1. The double sequence $x$ is P-convergent to $L$ if and only if every subsequence of $x$ is $\mathrm{P}$-convergent to $L$.

3. Main results. The next result is a "Steinhaus-type" theorem, so named because of its similarity to the Steinhaus theorem in [9] quoted in the introduction.

THEOREM 3.1. If $A$ is an RH-regular matrix, then there exists a bounded double sequence $x$ consisting only of 0 's and 1's which is not A-summable.

Proof. Let $m_{i}, n_{j}, k_{i}$, and $l_{j}$ be increasing index sequences which we define as follows:

Let $k_{0}:=l_{0}:=-1$ and choose $m_{0}$ and $n_{0}$ such that $m_{0}, n_{0}>B$, where $B$ is defined by $\mathrm{RH}_{6}$ and $\mathrm{RH}_{2}$ to imply

$$
\left|\sum_{k, l=0}^{\infty, \infty} a_{m_{0}, n_{0}, k, l}\right|>\frac{1}{4},
$$

whenever $m_{0}, n_{0}>B$.

Also, by $\mathrm{RH}_{1}, \mathrm{RH}_{3}, \mathrm{RH}_{4}$, and $\mathrm{RH}_{5}$ we choose $k_{1}>k_{0}$ and $l_{1}>l_{0}$ such that 


$$
\begin{gathered}
\left|\sum_{k<k_{1}, l<l_{1}} a_{m_{0}, n_{0}, k, l}\right|>1-\frac{1}{4}, \\
\sum_{k \geq k_{1}, l \geq l_{1}}\left|a_{m_{0}, n_{0}, k, l}\right|<\frac{1}{4}, \\
\sum_{k \geq k_{1}, l<l_{1}}\left|a_{m_{0}, n_{0}, k, l}\right|<\frac{1}{4}, \\
\sum_{k<k_{1}, l \geq l_{1}}\left|a_{m_{0}, n_{0}, k, l}\right|<\frac{1}{4} .
\end{gathered}
$$

Next use $\mathrm{RH}_{1}, \mathrm{RH}_{2}, \mathrm{RH}_{3}$, and $\mathrm{RH}_{4}$ to choose $m_{1}>m_{0}$ and $n_{1}>n_{0}$ such that

$$
\begin{aligned}
\sum_{k<k_{1}, l<l_{1}}\left|a_{m_{1}, n_{1}, k, l}\right| & <\frac{1}{9}, \\
\sum_{k \leq k_{1}, l \geq l_{1}}\left|a_{m_{1}, n_{1}, k, l}\right| & <\frac{1}{9}, \\
\sum_{k \geq k_{1}, l \leq l_{1}}\left|a_{m_{1}, n_{1}, k, l}\right| & <\frac{1}{9}, \\
\left|\sum_{k, l=0}^{\infty, \infty} a_{m_{1}, n_{1}, k, l}\right| & >1-\frac{1}{9} .
\end{aligned}
$$

These inequalities imply

$$
\sum_{k>k_{1}, l>l_{1}}\left|a_{m_{1}, n_{1}, k, l}\right|>1-\frac{4}{9},
$$

because

$$
\begin{aligned}
\left|\sum_{k>k_{1}, l>l_{1}}\right| a_{m_{1}, n_{1}, k, l}|| \geq & -\sum_{k \leq k_{1}, l \leq l_{1}}\left|a_{m_{1}, n_{1}, k, l}\right|+1-\frac{1}{9} \\
& -\sum_{k \geq k_{1}, l \leq l_{1}}\left|a_{m_{1}, n_{1}, k, l}\right| \\
& -\sum_{k \leq k_{1}, l>l_{1}}\left|a_{m_{1}, n_{1}, k, l}\right| .
\end{aligned}
$$

We now choose $k_{2}>k_{1}$ and $l_{2}>l_{1}$ such that

$$
\begin{gathered}
\left|\sum_{k_{1}<k<k_{2}, l_{1}<l<l_{2}} a_{m_{1}, n_{1}, k, l}\right|>1-\frac{4}{9}, \\
\sum_{k \geq k_{2}, l \geq l_{2}}\left|a_{m_{1}, n_{1}, k, l}\right|<\frac{1}{9}, \\
\sum_{k_{1}<k \leq k_{2}, l \geq l_{2}}\left|a_{m_{1}, n_{1}, k, l}\right|<\frac{1}{9}, \\
\sum_{k \geq k_{2}, l_{1}<l<l_{2}}\left|a_{m_{1}, n_{1}, k, l}\right|<\frac{1}{9} .
\end{gathered}
$$


In general, having

$$
\begin{array}{rr}
m_{0}<\cdots<m_{i-1}, & k_{0}<\cdots<k_{i-1}<k_{i}, \\
n_{0}<\cdots<n_{j-1}, & l_{0}<\cdots<l_{j-1}<l_{j},
\end{array}
$$

we choose $m_{i}>m_{i-1}$ and $n_{j}>n_{j-1}$ such that by $\mathrm{RH}_{1}$

$$
\sum_{k \leq k_{i}, l \leq l_{j}}\left|a_{m_{i}, n_{j}, k, l}\right|<\frac{1}{(i+2)(j+2)},
$$

and by $\mathrm{RH}_{3}, \mathrm{RH}_{4}$

$$
\begin{aligned}
& \sum_{k \leq k_{i}, l>l_{j}}\left|a_{m_{i}, n_{j}, k, l}\right|<\frac{1}{(i+2)(j+2)}, \\
& \sum_{k \geq k_{i}, l \leq l_{j}}\left|a_{m_{i}, n_{j}, k, l}\right|<\frac{1}{(i+2)(j+2)} .
\end{aligned}
$$

In addition, by $\mathrm{RH}_{2}$

$$
\left|\sum_{k, l=0}^{\infty, \infty} a_{m_{i}, n_{j}, k, l}\right|>1-\frac{1}{(i+2)(j+2)}
$$

so

$$
\sum_{k>k_{i}, l>l_{j}}\left|a_{m_{i}, n_{j}, k, l}\right|>1-\frac{4}{(i+2)(j+2)} .
$$

We now choose $k_{i+1}>k_{i}$ and $l_{j+1}>l_{j}$ such that

$$
\begin{aligned}
\sum_{k_{i}<k<k_{i+1}, l_{j}<l<l_{j+1}} a_{m_{i}, n_{j}, k, l} \mid & >1-\frac{4}{(i+2)(j+2)}, \\
\sum_{k \geq k_{i+1}, l \geq l_{j+1}}\left|a_{m_{i}, n_{j}, k, l}\right| & <\frac{1}{(i+2)(j+2)}, \\
\sum_{k_{i}<k<k_{i+1}, l \geq l_{j+1}}\left|a_{m_{i}, n_{j}, k, l}\right| & <\frac{1}{(i+2)(j+2)}, \\
\sum_{k \geq k_{i+1}, l_{j}<l<l_{j+1}}\left|a_{m_{i}, n_{j}, k, l}\right| & <\frac{1}{(i+2)(j+2)} .
\end{aligned}
$$

Define $x$ as follows:

$$
x_{k, l}= \begin{cases}1, & \text { if } k_{2 p}<k<k_{2 p+1} \text { and } l_{2 t}<l<l_{2 t+1} \text { for } p, t=0,1,2, \ldots \\ 0, & \text { otherwise }\end{cases}
$$


Let us label and partition $(A X)_{m_{i}, n_{j}}$ as follows:

$$
\begin{aligned}
(A X)_{m_{i}, n_{j}}= & \sum_{0 \leq k \leq k_{i}, 0 \leq l \leq l_{j}}^{\alpha_{1}}+\sum_{0 \leq k \leq k_{i}, l_{j+1} \leq l}^{\alpha_{2}}+\sum_{k_{i+1} \leq k, l_{j+1} \leq l}^{\alpha_{3}} \\
& +\sum_{0 \leq l \leq l_{j}, k_{i+1} \leq k}^{\alpha_{4}}+\sum_{k_{i}<k<k_{i+1}, 0 \leq l \leq l_{j}}^{\alpha_{5}}+\sum_{l_{j}<l<l_{j+1}, 0 \leq k \leq k_{i}}^{\alpha_{6}} \\
& +\sum_{k_{i}<k<k_{i+1}, l_{j+1} \leq l}^{\alpha_{7}}+\sum_{l_{j}<l<l_{j+1}, k_{i+1} \leq k}^{\alpha_{9}}+\sum_{k_{i}<k<k_{i+1}, l_{j}<l<l_{j+1}}^{\alpha_{9}} a_{m_{i}, n_{j}, k, l} x_{k, l},
\end{aligned}
$$

where the general term $a_{m_{i}, n_{j}, k, l} x_{k, l}$ is the same for each of the nine sums. Note that,

$$
\begin{aligned}
& \left|\alpha_{4}+\alpha_{5}\right| \leq \frac{1}{(i+2)(j+2)}, \\
& \left|\alpha_{2}+\alpha_{6}\right| \leq \frac{1}{(i+2)(j+2)} .
\end{aligned}
$$

CASE 1. If $i$ and $j$ are even, then

$$
\left|(A X)_{m_{i}, n_{j}}\right|>1-\frac{1}{(i+2)(j+2)}-\left|\alpha_{1}\right|-\cdots-\left|\alpha_{8}\right|>1-\frac{7}{(i+2)(j+2)},
$$

and the last expression has P-limit 1.

CASE 2. If at least one of $i$ and $j$ is odd, then $\alpha_{9}=0$ and

$$
\left|(A X)_{m_{i}, n_{j}}\right| \leq\left|\alpha_{1}\right|+\left|\alpha_{2}\right|+\cdots+\left|\alpha_{8}\right| \leq \frac{6}{(i+2)(j+2)},
$$

and the last expression of (3.17) has P-limit 0. Thus the P-limit of $(A X)_{m, n}$ does not exist, and we have shown that an RH-regular matrix $A$ cannot sum every double sequence, of 0's and 1's.

As with the original Steinhaus Theorem [9], we can state the following as an immediate consequence of Theorem 3.1.

COROLLARY 3.1. If $A$ is an RH-regular matrix, then A cannot sum every bounded double sequence.

The next result is a "Buck-type" theorem.

THEOREM 3.2. The bounded double complex sequence $x$ is $\mathrm{P}$-convergent if and only if there exists an RH-regular matrix A such that A sums every subsequence of $x$.

Proof. Since every subsequence of a P-convergent sequence $x$ is bounded and P-convergent, and $A$ is an RH-regular matrix, then for such an $x$ there exists an RH-regular matrix $A$ such that $S^{\prime \prime}\{x\} \subseteq C_{A}^{\prime \prime}$.

Conversely, we use an adaptation of Buck's proof [1] to show that if $A$ is any 
RH-regular matrix and $x \notin C^{\prime \prime}$ then there exists a subsequence $y \in S^{\prime \prime}\{x\}$ such that $A y \notin C^{\prime \prime}$.

Note that every subsequence of $x$ is bounded and $x \notin C^{\prime \prime}$, which implies that $x$ has at least two distinct Pringsheim limit points, say $\alpha$ and $\beta$. Thus there exist increasing index sequences $\left\{n_{j}\right\}$ and $\left\{k_{i}\right\}$ such that $\limsup x_{n_{i}, k_{i}}=\alpha$ and $\liminf x_{n_{i}, k_{i}}=\beta$ with $\alpha \neq \beta$.

Now define

$$
y=\frac{x-\beta}{\alpha-\beta}
$$

which yields $\lim \sup y_{n_{i}, k_{i}}=1$ and $\liminf y_{n_{i}, k_{i}}=0$. As a result there exist two disjoint pairs of index sequences $\left\{\bar{n}_{j}^{i}, \bar{k}_{j}^{i}\right\}$ and $\left\{v_{j}^{i}, k_{j}^{i}\right\}$ such that the sequences $\bar{y}_{1}$ and $\bar{y}_{2}$ constructed using $\left\{\bar{n}_{j}^{i}, \bar{k}_{j}^{i}\right\}$ and $\left\{v_{j}^{i}, k_{j}^{i}\right\}$, respectively, have P-limits 1 and 0 , respectively. Let

$$
y_{n, k}^{*}:= \begin{cases}1, & \text { if } n=\bar{n}_{j}^{i}, k=\bar{k}_{j}^{i}, \\ 0, & \text { if } n=v_{j}^{i}, k=k_{j}^{i}, \\ y, & \text { otherwise. }\end{cases}
$$

Hence, $\left\{y_{n, k}^{*}\right\}$ contains a subsequence $\left\{\bar{y}_{n, k}^{*}\right\}$ with infinitely many 0 's and 1's, along its diagonal. This implies that $S^{\prime \prime}\left\{\bar{y}^{*}\right\}$ contains all sequences of 0's and 1's. Thus by Theorem 3.1, there exists $\tilde{y}^{*} \in S^{\prime \prime}\left\{\bar{y}^{*}\right\}$ such that $A \tilde{y}^{*} \notin C^{\prime \prime}$. Also, P-lim $\left(y-y^{*}\right)_{i, j}=$ 0 . We now select a subsequence $\left\{\tilde{y}_{i, j}\right\}$ of $\left\{y_{i, j}\right\}$ with terms satisfying $\lim \sup _{i} y_{n_{i}, k_{i}}=1$ and liminf ${ }_{i} y_{n_{i}, k_{i}}=0$ corresponding to the 0 's and 1's, respectively of $\left\{\tilde{y}_{i, j}^{*}\right\}$. Therefore P-lim $\left(\tilde{y}-\tilde{y}^{*}\right)_{i, j}=0$ and $\tilde{y}_{i, j}-\tilde{y}_{i, j}^{*}$ is bounded. By the linearity and regularity of $A, A\left(\tilde{y}-\tilde{y}^{*}\right)_{i, j}=(A \tilde{y})_{i, j}-\left(A \tilde{y}^{*}\right)_{i, j}$ and P-lim $A\left(\tilde{y}-\tilde{y}^{*}\right)_{i, j}=0$. Now since $A \tilde{y}^{*} \notin C^{\prime \prime}$, it follows that $A \tilde{y} \notin C^{\prime \prime}$; and since $\tilde{y}=\bar{x}-\beta / \alpha-\beta$, we have $A \tilde{x} \notin C^{\prime \prime}$.

ACKNOWLEDGEMENT. This paper is based on the author's doctoral dissertation, written under the supervision of Prof. J. A. Fridy at Kent State University. I am extremely grateful to my advisor Prof. Fridy for his encouragement and advice.

\section{REFERENCES}

[1] R. C. Buck, A note on subsequences, Bull. Amer. Math. Soc. 49 (1943), 898-899. MR 5,117b. Zbl 060.15802 .

[2] H. J. Hamilton, Transformations of multiple sequences, Duke Math. J. 2 (1936), 29-60. Zbl 013.30301.

[3] _ C Change of dimension in sequence transformations, Duke Math. J. 4 (1938), 341-342. Zbl 019.05901.

[4] _ A generalization of multiple sequence transformations, Duke Math. J. 4 (1938), 343-358. Zbl 019.05902.

[5] _ Preservation of partial limits in multiple sequence transformations, Duke Math. J. 5 (1939), 293-297. Zbl 021.22103.

[6] G. H. Hardy, Divergent Series, Oxford at Clarendon Press, London, 1949. MR 11,25a. Zbl 032.05801.

[7] A. Pringsheim, Zur theorie de zweifach unendlichen Zahlenfolgen, Mathematische Annalen 53 (1900), 289-321. 
[8] G. M. Robison, Divergent double sequences and series, Trans. Amer. Math. Soc. 28 (1926), 50-73.

[9] H. Steinhaus, Remarks on the generalization of the idea of limit, Praco Matematyczne Fizyczne 22 (1911), 121-134 (Polish).

PATTERSON: DEPARTMENT OF MATHEMATICS AND COMPUTER SCIENCE, DUQUESNE UNIVERSITY, 440 College Hall, PitTsBurgh, PA 15282, USA

E-mail address: pattersr@mathcs.duq.edu 


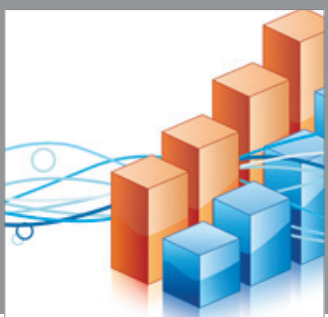

Advances in

Operations Research

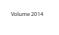

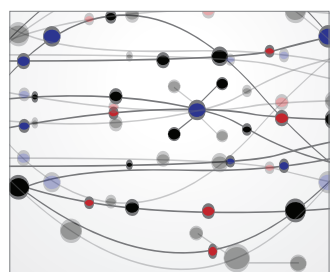

\section{The Scientific} World Journal
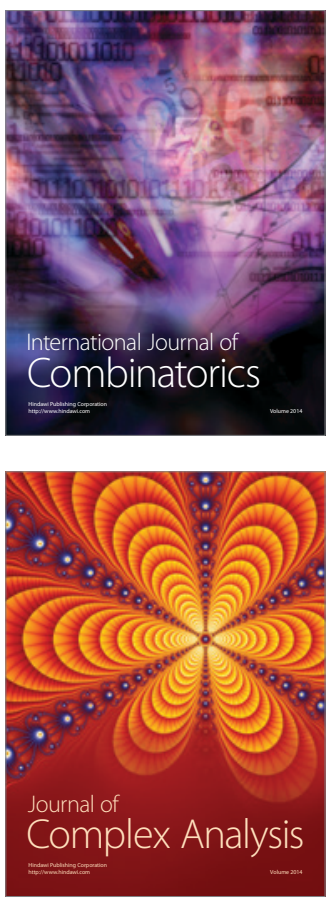

International Journal of

Mathematics and

Mathematical

Sciences
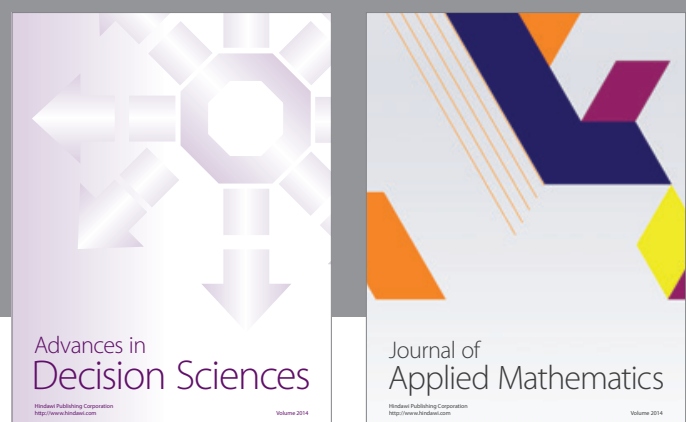

Journal of

Applied Mathematics
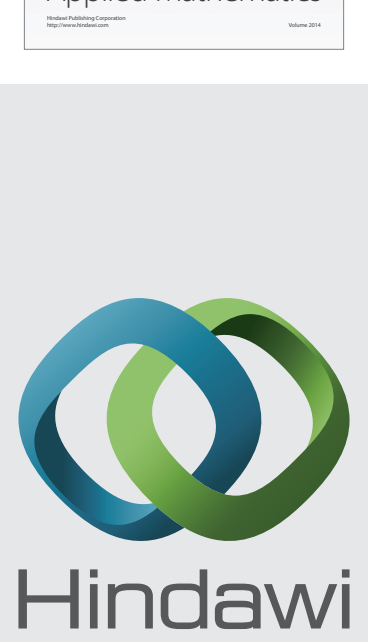

Submit your manuscripts at http://www.hindawi.com
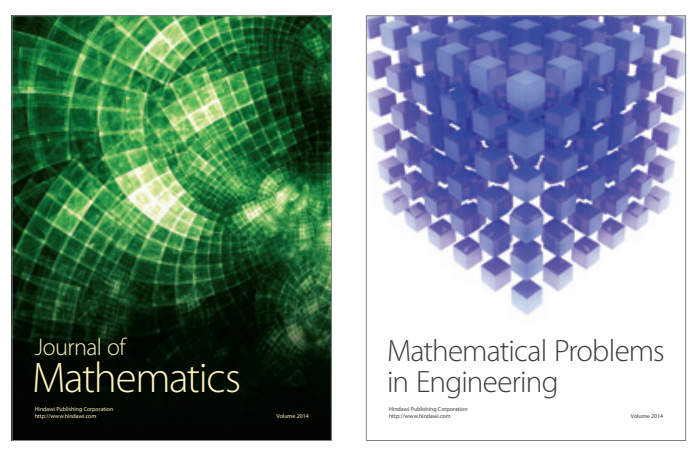

Mathematical Problems in Engineering
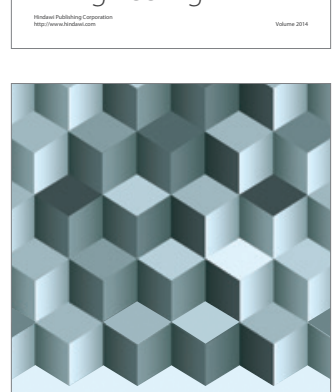

Journal of

Function Spaces
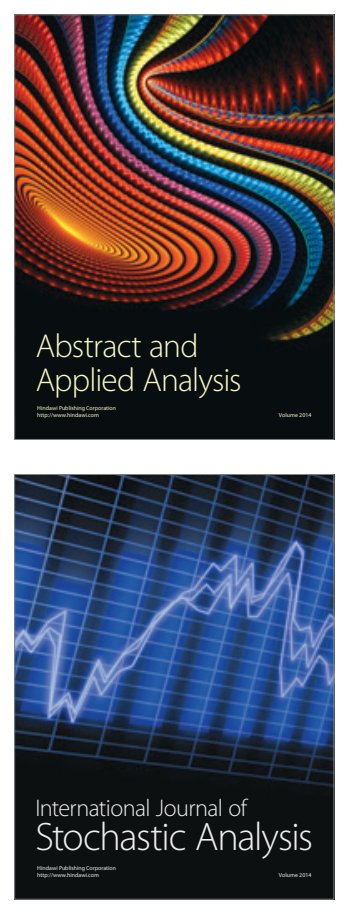

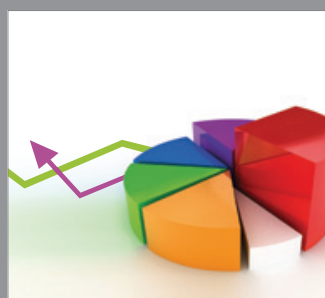

ournal of

Probability and Statistics

Promensencen
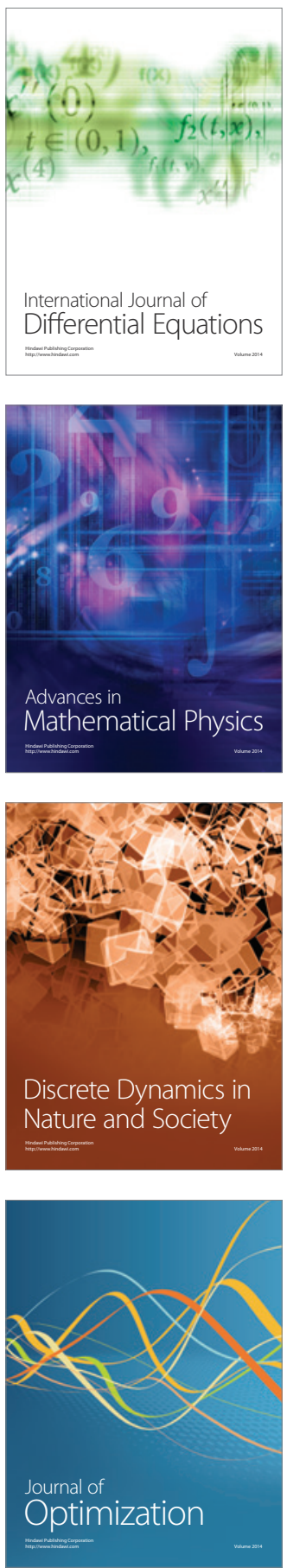